\title{
Orphans to the rescue: orphan G-protein coupled receptors as new antidepressant targets
}

\author{
Paul R. Albert, PhD
}

Are orphan G-protein coupled receptors (oGPRs) the next frontier in psychiatric drug targets? Several recent papers suggest that this may finally be the case. G-protein coupled receptors are the target of a large proportion of approved and emerging therapeutic compounds, including for psychiatric illnesses. ${ }^{1,2}$ The oGPRs are receptors for which the endogenous ligand is not known. The first "orphan" receptor human genomic clone, G-21, was discovered in 1987 using lowstringency hybridization with the $\beta 2$-adrenergic receptor cDNA. ${ }^{3}$ G-21 and its rat homologue were subsequently identified as the serotonin-1A (5HT1A) receptor, based on ligand binding studies. ${ }^{4,5}$ The same year, the dopamine-D2 receptor was also identified in this way. ${ }^{6}$ Subsequently, many other GPR-like genes were cloned using polymerase chain reactionbased approaches, but for many their identity was unknown. ${ }^{7,8}$ Since endogenous ligands for these receptors remained unidentified they were classified as oGPRs. "Deorphanization" is the discovery of these endogenous compounds $^{8}$ and has led to the identification of several new families of receptors. These include GPRs for nonclassical ligands, including lipids, ${ }^{9}$ ions, trace amines and adhesion molecules. ${ }^{10}$ Recently, oGPRs have been associated with psychiatric illnesses, including major depression, and with responsiveness to antidepressants. ${ }^{11,12}$ These emerging findings highlight their potential importance in susceptibility and treatment of mental illness. Targeting oGPRs may provide novel pharmacological agents for improved treatment of mental illnesses.

\section{Roles of oGPRs in depression}

A variety of approaches have implicated oGPRs in depression and antidepressant activity. Typically, gene knockout or viral knockdown of oGPRs (e.g., small interfering RNA) has been used to address their biological function in animal models because of the paucity of specific high-affinity ligands for these receptors. Where available, ligands for oGPRs have also been used in rodent models to test for antidepressant activity. Genomic, proteomic or candidate gene analyses of human tissues have recently revealed changes in oGPR expression levels in blood or postmortem brain from depressed individ- uals compared with nonpsychiatric controls. Together, these approaches have elucidated several oGPRs as potential biomarkers or drug targets for treatment of major depression. Some recent examples are presented here (Box 1).

\section{GPR56, an adhesion GPR, enhances antidepressant response}

Recently, Belzeaux and colleagues ${ }^{12}$ screened for blood biomarkers for response to treatment with the serotonin/noradrenaline reuptake inhibitor duloxetine. They found that of 42 positive probes, the adhesion receptor GPR56 showed the greatest increase (19\%) compared with nonresponders. This finding was replicated in 2 smaller cohorts receiving either the serotonin reuptake inhibitor citalopram or various antidepressants. In a chronic stress mouse model of depression, responders to fluoxetine showed increased GPR56 expression in blood and prefrontal cortex (PFC). Genetically induced depletion of GPR56 in mouse PFC resulted in a mild depression-like phenotype and resistance to fluoxetine treatment. Conversely, microinjection of GPR56 agonist peptide in the PFC had an acute antidepressant effect, suggesting GPR56 as a potential target to develop novel antidepressants. However, it would be important to first test whether GPR56 activation has a chronic antidepressant effect.

\section{GPR158: a pro-depressant glutamate-related GPR}

As a second example, Sutton and colleagues ${ }^{11}$ screened specifically for oGPR expression changes in a chronic stress mouse model of depression compared with unstressed mice. They showed that the PFC expresses a wide variety of oGPRs, with GPR158 being among the most abundant. GPR158 is structurally related to metabotropic glutamate receptors, except that it lacks the extracellular "Venus fly trap" glutamate binding domain. ${ }^{13}$ GPR158 was strongly induced in PFC glutamate neurons and in blood, probably via stress-induced glucocorticoids. It was also increased in the dorsolateral PFC of depressed compared with control human brains. Overexpression of GPR158 in the mouse PFC mimicked the stress-induced depressive phenotype, while GPR158 knockout mice showed reduced

Correspondence to: P. Albert, Ottawa Health Research Institute (Neuroscience), University of Ottawa, 451 Smyth Road, Ottawa, Ont K1H 8M5; palbert@uottawa.ca

DOI: $10.1503 / j p n .200149$ 
Box 1: Highlighted oGPRs implicated in major depression*

\begin{tabular}{lccccc}
\hline Orphan & GPR Family & Effect & Species studied & Endogenous ligand & Synthetic \\
\hline GPR56 & Adhesion & Anti Dep; Enh AD & H/R & Collagen III/TG2 & Peptide Ag; Non-peptide Antag \\
GPR158 & Adhesion, mGluR-like & Pro Dep & H/R & Proteoglycan GPC4 & None \\
GPR39 & Ghrelin-like & Anti Dep; Enh AD & $\mathrm{H} / \mathrm{R}$ & Zn $^{2+}$ & TC-G 1008 PAM \\
GPR88 & 5-HT1B-like & Pro Dep (PD) & $\mathrm{R}$ & Unknown & RTI-13951-33 Ag \\
GPR160 & Amine-like & Pro Pain & $\mathrm{R}$ & CART & Peptide CART Ag \\
\hline
\end{tabular}

5-HT1B = serotonin 1B; Ag = agonist; Antag = antagonist; CART = cocaine/amphetamine-regulated transcript; GPR = G-protein coupled receptors; mGluR = metabotropic glutamate receptors; oGPR = orphaned G-protein coupled receptors; PAM = positive allosteric modulator; $\mathrm{TG}=$ transglutaminase .

*Shown for the highlighted oGPRs are the receptor family; the effect of the receptor (Pro or Anti) on depression (Dep) alone or in Parkinson disease (PD); on pain; and on enhancing (Enh) antidepressant response (AD); the species studied (human $\mathrm{H}$, rodent $\mathrm{R}$ ), and endogenous or exogenous ligands.

depressive behaviour and enhanced PFC glutamatergic activity. Thus, inhibition of GPR158 expression or activity may have antidepressant activity, opposite to the role of GPR56.

\section{GPR39: a zinc receptor that mediates antidepressant response}

Another oGPR implicated in depression and antidepressant response is the ghrelin-like zinc receptor GPR39. Structurally, GPR39 is in the ghrelin receptor subfamily; however, its peptide ligand remains elusive. ${ }^{8}$ Although GPR39 is activated by zinc, zinc has many targets thus limiting its usefulness in identifying the function of this receptor. Knockout of GPR39 in mice results in anxiety- and depression-like behaviours and resistance to monoamine-enhancing antidepressants, but not to ketamine. . $^{14,15}$ GPR39 appears to activate cyclic adenosine monophosphate (cAMP) signalling, and cAMP response element binding protein (CREB)/brain-derived neurotrophic factor (BDNF) levels were reduced in the frontal cortex and hippocampus of GPR39 knockout mice. In human studies, levels of GPR39 protein were reduced in the hippocampus and cortex of suicide compared with control brains. ${ }^{16}$ These studies implicate GPR39 in depression and resistance to antidepressant treatment, although its regulation and role needs to be studied in a clinical population.

\section{oGPRs as depression biomarkers}

Although GPR56 was identified using a biomarker approach, ${ }^{12}$ the small enrichment in the treatment response group limits its utility as a prognostic peripheral biomarker. However, GPR56 expression did not differ between antidepressant responders and nonresponders at admission. It was induced only after 8 weeks of treatment, and more robustly at 30 weeks. Thus, GPR56 cannot be used to predict antidepressant response, but appears to be induced by selective serotonin reuptake inhibitors and serotonin-norepinephrine reuptake inhibitors. For GPR158, the association of its blood levels with depression in humans or mouse models appears to mainly reflect chronic increases in serum glucocorticoids induced by stress. ${ }^{11}$ Glucocorticoids enter the brain and regulate gene expression via glucocorticoid receptors in both brain and blood with correlated activity. Indeed, many biomarkers simply reflect chronic glucocorticoid load ${ }^{17,18}$ and are thus biomarkers of chronic stress rather than depression per se. ${ }^{19}$ In a complementary way, genetic polymorphisms in oGPR genes may also provide blood biomarkers for chronic stress. However, some may be specific for subtypes of depression. For example, polymorphisms that disrupt the X-chromosome gene GPR50 have been associated with bipolar depression in a small study in women. ${ }^{20}$ However, this needs further replication in larger samples, and it is unlikely that single gene polymorphisms will be predictive.

\section{Targeting oGPRs to develop new antidepressants}

Because ligands for oGPRs are often lacking, the mechanisms of oGPR actions have been difficult to assess. GPR56 is an adhesion receptor that binds matrix proteins collagen type III and transglutaminase and is implicated in cortical development. ${ }^{10}$ Adhesion receptors are activated by self-cleavage of an $\mathrm{N}$ terminal tethered peptide upon binding to synaptic or extracellular matrix proteins. For example, GPR56 binds to collagen III and transglutaminase $2 .^{10}$ Thus, these receptor-derived tethered peptides can be used as agonists. However, as peptides they have limited stability, brain permeability, affinity and specificity since they are not constrained at the receptor as is the endogenous peptide. Recently, drug screens have begun to identify nonpeptide ligands for GPR56 including high-affinity antagonists, suggesting that this class of oGPRs may yield pharmacologically useful compounds. ${ }^{21}$

Like GPR56, GPR158 appears to recognize an extracellular matrix component, heparan sulfate proteoglycan GPC4. As an adhesion receptor, GPR158 has been implicated in synaptic contacts between hippocampal mossy fibres and CA3 pyramidal neurons. ${ }^{22}$ Instead of a glutamate binding site, GPR158 contains calcium-binding epidermal growth factor (EGF)-like and Leu-rich N-terminal domains that may mediate these interactions. ${ }^{13}$ It constitutively recruits RGS7-Gb5 to the membrane to inactivate signalling of inhibitory G-proteins $(\mathrm{Gi} / \mathrm{Go}){ }^{13,23}$ GPR158 also prevents cAMP-mediated inactivation of Kv4.2 potassium channels, reducing PFC pyramidal neuronal activity. ${ }^{24}$ Thus, stress-induced induction of GPR158 mediates inactivation of PFC activity. It may also affect synapses to cortical neurons as seen in the hippocampus, but this has not been reported.

In the case of GPR39, a screen of Chinese hamster ovary cells transfected with human GPR39 led to the discovery of a potent positive allosteric modulator, GPR39-C3/TC-G 1008. ${ }^{25}$ TC-G 1008 is highly specific to enhance zinc activation of GPR39, but also binds to 5-HT1A receptors. ${ }^{26}$ Importantly, TC-G 1008 shows persistent antidepressant activity in mice. ${ }^{27}$ While further ligand optimization for receptor specificity, kinetics and toxicology is needed, GPR39 agonists may provide novel antidepressant treatments. 


\section{The future for oGPRs in psychiatry}

Identifying the roles of specific oGPRs in mental illness has uncovered additional leads to those discussed here, but many oGPRs remain to be studied. For example, GPR88, which is strongly expressed in the striatum, has been recently implicated in depression-like behaviour in a rat model of Parkinson disease. ${ }^{28}$ However, high-affinity ligands for GPR88 have yet to be identified. ${ }^{29}$ The recent brain expression mapping of 78 oGPR genes ${ }^{30}$ will provide a useful resource to begin to address the roles of oGPRs in neuropsychiatric diseases. Ongoing deorphanization efforts may reveal new drug targets. A case in point, GPR160 has recently been shown to be the CART (cocaine/amphetamineregulated transcript) receptor, with implications for pain and drug addiction. ${ }^{31}$ Agonists of GPR56 and GPR39 show antidepressant activity in mouse models of depression, with potential as novel antidepressant or augmentation therapies in human depression. On the other hand, antagonists of GPR158 may promote resilience to depression, as in mouse models. However, these oGPRs are widely distributed in the brain and in development, which may lead to unexpected or adverse effects. While their functions can be parsed using knockout approaches in mice, ultimately ligand testing in humans will determine feasibility, safety and efficacy of targeting these receptors. Because the time from deorphanization to drug discovery can exceed 10 years, ${ }^{8}$ these orphans, while promising, will need time to advance in psychiatric treatments. Nevertheless, accumulating evidence on critical roles in psychiatry for this pharmacologically rich family of proteins suggests that these orphans may help rescue the dearth of new lead compounds for treatment of mental illness. ${ }^{32}$

Affiliations: From the Ottawa Hospital Research Institute (Neuroscience), UOttawa Brain and Mind Research Institute, Ottawa, Ont.

Competing interests: None declared.

\section{References}

1. Vortherms TA, Roth BL. Receptorome screening for CNS drug discovery. IDrugs 2005;8:491-6.

2. Seyedabadi $M$, Ghahremani $M H$, Albert PR. Biased signaling of $G$ protein coupled receptors (GPCRs): molecular determinants of GPCR/transducer selectivity and therapeutic potential. Pharmacol Ther 2019;200:148-78.

3. Kobilka BK, Frielle T, Collins S, et al. An intronless gene encoding a potential member of the family of receptors coupled to guanine nucleotide regulatory proteins. Nature 1987;329:75-9.

4. Fargin A, Raymond JR, Lohse MJ, et al. The genomic clone G-21 which resembles a beta-adrenergic receptor sequence encodes the 5-HT1A receptor. Nature 1988;335:358-60.

5. Albert PR, Zhou QY, Van Tol HH, et al. Cloning, functional expression, and mRNA tissue distribution of the rat 5-hydroxytryptamine1A receptor gene. J Biol Chem 1990;265:5825-32.

6. Bunzow JR, Van Tol HH, Grandy DK, et al. Cloning and expression of a rat D2 dopamine receptor cDNA Nature 1988;336:783-7.

7. Fredriksson R, Lagerstrom MC, Lundin L-G, et al. The G-proteincoupled receptors in the human genome form five main families. Phylogenetic analysis, paralogon groups, and fingerprints. Mol Pharmacol 2003;63:1256-72.

8. Civelli O, Reinscheid RK, Zhang Y, et al. G protein-coupled receptor deorphanizations. Annu Rev Pharmacol Toxicol 2013;53:127-46.
9. Hudson BD, Smith N, Milligan G. Experimental challenges to targeting poorly characterized GPCRs: uncovering the therapeutic potential for free fatty acid receptors. Adv Pharmacol 2011;62:175-218.

10. Hamann J, Aust G, Arac D, et al. International union of basic and clinical pharmacology. XCIV. Adhesion G protein-coupled receptors. Pharmacol Rev 2015;67:338-67.

11. Sutton LP, Orlandi C, Song C, et al. Orphan receptor GPR158 controls stress-induced depression. eLife 2018;7:e33273.

12. Belzeaux R, Gorgievski V, Fiori LM, et al. GPR56/ADGRG1 is associated with response to antidepressant treatment. Nat Commun 2020;11:1635

13. Orlandi C, Posokhova E, Masuho I, et al. GPR158/179 regulate G protein signaling by controlling localization and activity of the RGS7 complexes. J Cell Biol 2012;197:711-9.

14. Mlyniec K, Budziszewska B, Holst B, et al. GPR39 (zinc receptor) knockout mice exhibit depression-like behavior and CREB/BDNF down-regulation in the hippocampus. Int J Neuropsychopharmacol 2014;18:pyu002.

15. Mlyniec K, Gawel M, Nowak G. Study of antidepressant drugs in GPR39 (zinc receptor(-)/(-)) knockout mice, showing no effect of conventional antidepressants, but effectiveness of NMDA antagonists. Behav Brain Res 2015;287:135-8.

16. Mlyniec K, Doboszewska U, Szewczyk B, et al. The involvement of the GPR39-Zn(2+)-sensing receptor in the pathophysiology of depression. Studies in rodent models and suicide victims. Neuropharmacology 2014;79:290-7.

17. Belzeaux R, Lin R, Ju C, et al. Transcriptomic and epigenomic biomarkers of antidepressant response. J Affect Disord 2018;233:36-44.

18. Bakusic J, Schaufeli W, Claes S, et al. Stress, burnout and depression a systematic review on DNA methylation mechanisms. J Psychosom Res 2017;92:34-44.

19. Juruena MF, Bocharova M, Agustini B, et al. Atypical depression and non-atypical depression: Is HPA axis function a biomarker? A systematic review. J Affect Disord 2018;233:45-67.

20. Thomson PA, Wray NR, Thomson AM, et al. Sex-specific association between bipolar affective disorder in women and GPR50, an Xlinked orphan G protein-coupled receptor. Mol Psychiatry 2005;10: 470-8.

21. Stoveken HM, Larsen SD, Smrcka AV, et al. Gedunin- and khivorinderivatives are small-molecule partial agonists for adhesion $\mathrm{G}$ proteincoupled receptors GPR56/ADGRG1 and GPR114/ADGRG5. Mol Pharmacol 2018;93:477-88.

22. Condomitti G, Wierda KD, Schroeder A, et al. An input-specific orphan receptor GPR158-HSPG interaction organizes hippocampal mossy fiber-CA3 synapses. Neuron 2018;100:201-15 e9.

23. Hajj M, De Vita T, Vol C, et al. Nonclassical ligand-independent regulation of go protein by an orphan class C G-protein-coupled receptor. Mol Pharmacol 2019;96:233-46.

24. Song C, Orlandi C, Sutton LP, et al. The signaling proteins GPR158 and RGS7 modulate excitability of L2/3 pyramidal neurons and control A-type potassium channel in the prelimbic cortex. J Biol Chem 2019;294:13145-57.

25. Peukert S, Hughes R, Nunez J, et al. Discovery of 2-pyridylpyrimidines as the first orally bioavailable GPR39 agonists. ACS Med Chem Lett 2014;5:1114-8.

26. Sato S, Huang XP, Kroeze WK, et al. Discovery and characterization of novel GPR39 agonists allosterically modulated by zinc. Mol Pharmacol 2016;90:726-37.

27. Starowicz G, Jarosz M, Frföckiewicz E, et al. Long-lasting antidepressant-like activity of the GPR39 zinc receptor agonist TC-G 1008. J Affect Disord 2019;245:325-34.

28. Galet B, Ingallinesi M, Pegon J, et al. G-protein coupled receptor 88 knockdown in the associative striatum reduces psychiatric symptoms in a translational male rat model of Parkinson disease. J Psychiatry Neurosci 2020;45:190171.

29. Ye N, Li B, Mao Q, et al. Orphan receptor GPR88 as an emerging neurotherapeutic target. ACS Chem Neurosci 2019;10:190-200.

30. Ehrlich AT, Maroteaux G, Robe A, et al. Expression map of 78 brainexpressed mouse orphan GPCRs provides a translational resource for neuropsychiatric research. Commun Biol 2018;1:102.

31. Yosten GL, Harada CM, Haddock C, et al. GPR160 de-orphanization reveals critical roles in neuropathic pain in rodents. J Clin Invest 2020;130:2587-2592.

32. Blier P. The well of novel antidepressants: running dry. J Psychiatry Neurosci 2010;35:219-20. 